\title{
Bone loss during neoadjuvant/adjuvant chemotherapy for early stage breast cancer: A retrospective cohort study
}

\author{
CHRISTIAN TANG AXELSEN ${ }^{1}$, ANDERS BONDE JENSEN ${ }^{1,2}$, \\ ERIK HUGGER JAKOBSEN ${ }^{3}$ and TROELS BECHMANN ${ }^{3,4}$
}

\author{
${ }^{1}$ Institute of Clinical Medicine, Aarhus University; ${ }^{2}$ Department of Oncology, Aarhus University Hospital, 8000 Aarhus; \\ ${ }^{3}$ Department of Oncology, Lillebaelt Hospital, 7100 Vejle; ${ }^{4}$ Department of Regional Health Research, \\ Faculty of Health Sciences, University of Southern Denmark, 5230 Odense, Denmark
}

Received September 30, 2017; Accepted January 9, 2018

DOI: $10.3892 / \mathrm{mco} .2018 .1615$

\begin{abstract}
The present study aimed to evaluate the extent of loss in bone mineral density (BMD) during neoadjuvant and adjuvant chemotherapy for early stage breast cancer. A retrospective cohort study was conducted to quantify the loss of BMD one year following the start of chemotherapy and to identify potential risk factors of excessive BMD loss. Based on DXA-scans prior to and one year following chemotherapy, the loss of BMD was evaluated in early stage breast cancer patients treated from January 2012 to December 2014. A total of 492 patients received either eight cycles of neoadjuvant or six cycles of adjuvant chemotherapy. The final analysis included 152 patients with two DXA-scans. The patients had a significant loss of BMD in the hip $\left[-0.0124 \mathrm{~g} / \mathrm{cm}^{2}(95 \%\right.$ confidence interval $(\mathrm{CI})-0.018 ;-0.007) \mathrm{P}<0.001]$ and in the lumbar spine [-0.029 g/ $/ \mathrm{cm}^{2}(95 \%$ CI: $\left.-0.036 ;-0.023) \mathrm{P}<0.001\right]$ corresponding to a change of $-1,3$ and $-2,9 \%$, respectively. Premenopausal women had a significant loss of BMD in the lumbar spine $-0.045 \mathrm{~g} / \mathrm{cm}^{2}$ equivalent to $-4.3 \%$, which was significantly increased compared with postmenopausal women $(\mathrm{P}<0.001)$ in the univariate analysis, whereas only a trend persisted in the multivariate analysis $(\mathrm{P}=0.60)$. There was no significant difference in BMD loss (lumbar spine $\mathrm{P}=0.176$ ) between patients receiving adjuvant and neoadjuvant chemotherapy. In conclusion, neoadjuvant and adjuvant chemotherapy is associated with significant BMD loss in both hip and lumbar spine. Furthermore, the results of the present study indicate that premenopausal women have a pronounced BMD loss in the lumbar spine. Further studies investigating osteoporosis prophylaxis in premenopausal patients are warranted.
\end{abstract}

Correspondence to: Dr Christian Tang Axelsen, Institute of Clinical Medicine, Aarhus University, Høegh-Guldbergs Gade 99b, 2. TH, 8000 Aarhus, Denmark

E-mail: c_tang1@hotmail.com

Key words: breast cancer, adjuvant chemotherapy, neoadjuvant chemotherapy, osteoporosis, bone mineral density, premenopausal

\section{Introduction}

Breast cancer (BC) is the most common cancer among women worldwide with 1.67 million new cases in 2012, and the incidence has until recently been continuously increasing (1). The 5-year relative survival has improved over time and is now around $85 \%$ (2), which is partly explained by earlier diagnosis and improved cancer treatment, including surgery, chemotherapy, radiation, HER2-tageted treatment, and endocrine therapy. In Denmark alone, $\geq 62,000$ women live with the potential side effects of breast cancer treatment (2). One important side effect is the increased bone turnover, the so called cancer treatment-induced bone loss (CTIBL) (3), which potentially may cause osteoporosis in the long run.

Osteoporosis is defined as a skeletal disorder compromising bone strength and leading to increased risk of fracture (4). Microarchitecture (quality) and bone density (quantity) are the two components of bone strength. For practicality the diagnosis is not based on microarchitecture but most often on measurements of the bone mineral density (BMD) by DXA scanning. The criterion of osteoporosis in postmenopausal women is a score of $2.5 \mathrm{SD}$ below the mean BMD of a young healthy woman (equivalent of a T-score <-2.5) (4). The diagnosis is based on the lowest BMD either at the lumbar spine or proximal femur (4).

So far, the literature has shown the highest extent of bone loss to take place within the first six months after start of chemotherapy $(5,6)$. The use of corticosteroids is a well-known risk factor of osteoporosis when administered low-dose over a long period of time (7). The effect is more uncertain when given as pulse doses in supportive care to prevent vomiting and nausea. Despite the large doses given over a relatively short time period, a recent study found no relation between prednisolone dose and BMD change (8). Actually, the BMD had increased four months after adjuvant chemotherapy, contrary to what other studies have shown (3,8-10).

Most studies on CTIBL address the effect in postmenopausal women, where chemotherapy itself induces bone loss $(11,12)$. Furthermore, standard adjuvant endocrine treatment in the form of aromatase inhibitors for five years increases the bone turnover 2-3 fold in postmenopausal women and decreases the BMD significantly due to the low 
levels of systemic oestrogen $(13,14)$. For premenopausal women chemotherapy-induced premature ovarian dysfunction is a long term risk factor of bone loss leading to increased risk of osteoporosis and thereby fractures $(5,10,13,15)$. Also, premenopausal patients with oestrogen receptor (ER) positive $\mathrm{BC}$ are likely to receive 10 years of treatment with tamoxifen resulting in increased bone turnover. Tamoxifen is able to preserve bone mass in postmenopausal women, whereas it may cause bone loss in premenopausal women (16-18).

The current guideline in Denmark recommends zoledronic acid as adjuvant therapy in postmenopausal women to reduce the risk of skeletal recurrence and improve survival (19). Zoledronic acid also reduces the risk of fractures and improves bone health over time by decreasing the osteoclast bone resorption activity and hence limiting CTIBL $(6,19)$. Since no effect of zoledronic acid on survival in premenopausal patients has been shown, it is not recommended in this subpopulation. Therefore the younger patients may be more prone to later development of osteoporosis (19).

We aimed to quantify bone loss and identify risk factors associated with increased bone loss during neoadjuvant/adjuvant chemotherapy. Menopausal status and neoadjuvant compared to adjuvant chemotherapy was of particular interest to elucidate the influence of extended chemotherapy and the amount of corticosteroids used in supportive care.

\section{Patients and methods}

Patients. This investigation was conducted as a retrospective cohort study including 492 patients with early stage BC, who received either adjuvant or neoadjuvant chemotherapy at the Department of Oncology, Vejle Hospital, Denmark between January 1, 2012 and December 31, 2014. A total of 340 patients were excluded from the final analysis as seen in the Consort diagram (Fig. 1). Most patients (316) were excluded due to a missing baseline DXA scan before the first cycle of chemotherapy or a missing follow-up DXA scan one year after the first DXA scan \pm 90 days. Eleven patients were diagnosed with osteoporosis before or at the first DXA scan and were excluded to avoid treatment directly influencing BMD. Thirteen patients had the first DXA scan more than 30 days after the first day of chemotherapy and were thus excluded. For the final analysis 152 patients were left.

Data was collected through the electronic patient record system after approval by the Danish Health and Medicines Authority and the Danish Data Protection Agency. According to Danish law this study did not require approval by the Ethics Committee (project-ID: S-20150139).

Treatment. The vast majority of the patients receiving adjuvant chemotherapy (69 out of 71), had three cycles of epirubicin $\left(90 \mathrm{mg} / \mathrm{m}^{2}, \mathrm{q} 3 \mathrm{w}\right)$ and cyclophosphamide $\left(600 \mathrm{mg} / \mathrm{m}^{2}, \mathrm{q} 3 \mathrm{w}\right)$ (EC) followed by three cycles of docetaxel $\left(100 \mathrm{mg} / \mathrm{m}^{2}, \mathrm{q} 3 \mathrm{w}\right)$. The remaining two patients had three and five cycles of chemotherapy, respectively. Seventy-two of the 81 patients receiving neoadjuvant chemotherapy had four cycles of paclitaxel $\left(80 \mathrm{mg} / \mathrm{m}^{2}, \mathrm{q} 1 \mathrm{w}\right.$, for 11 weeks) or docetaxel and four cycles of EC. The majority of the nine remaining patients received only six or seven cycles of chemotherapy due to side effects. The dose of chemotherapy was given according to national guidelines.

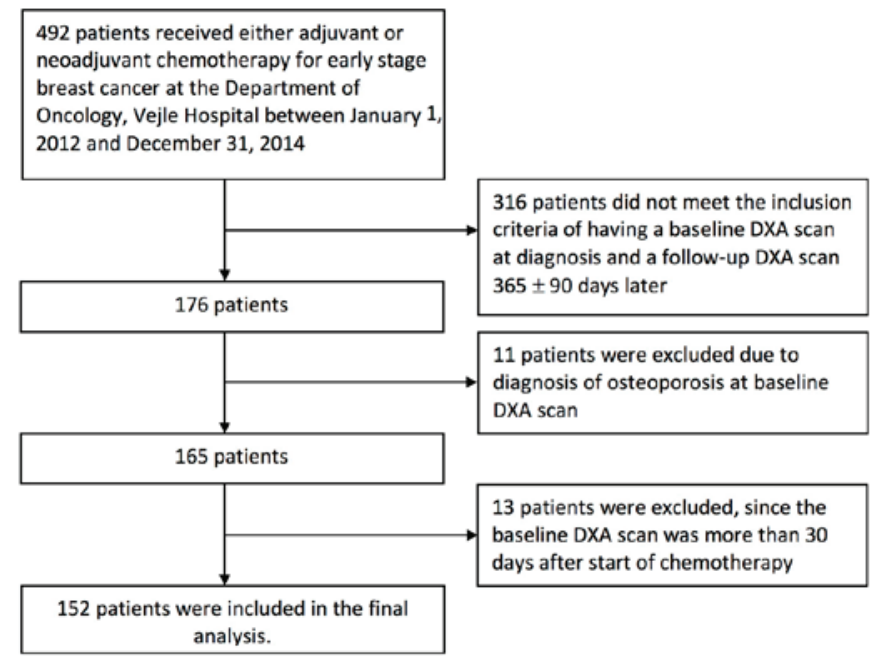

Figure 1. Consort diagram of inclusion of patients.

Prophylactic prednisolone against nausea, vomiting, and anaphylaxis was given in standard doses: $150 \mathrm{mg}$ prednisolone per cycle with EC, $300 \mathrm{mg}$ per cycle with docetaxel, and $100 \mathrm{mg}$ weekly with paclitaxel. In total, $1,350 \mathrm{mg}$ of prednisolone was given during standard adjuvant chemotherapy and $1,700 \mathrm{mg}$ during standard neoadjuvant chemotherapy.

Following neoadjuvant/adjuvant chemotherapy patients with ER-positive tumours started either tamoxifen or AI depending on the menopausal status at diagnosis. Adjuvant zoledronic acid was implemented in the guidelines at Vejle Hospital early 2014 and subsequently, postmenopausal patients were offered zoledronic acid $4 \mathrm{mg}$ every six months for a period of 4 years.

Clinical and histological data. Data on age, height, weight, smoking status, date of referral, type of surgery, chemotherapy, endocrine treatment, HER2-targeted treatment, and concurrent medicine were obtained from the patient's medical record. Pathology data, including histology type, HER2-status (defined as positive with immunohistochemistry $3+$ or $2+$ combined with fluorescence in situ hybridization ratio $\geq 2.0$ ), ER-status (defined positive in case of $1 \%$ or more ER positive tumour cells), and nodal status, were obtained from the patient's pathology records. BMI was calculated as weight in $\mathrm{kg}$ divided by the height in meters squared. BMI was categorized as less than 25 (underweight/normal), 25 to 30 (overweight) and more than 30 (obese) according to the World Health Organization criteria.

Outcome. T-score and BMD $\left(\mathrm{g} / \mathrm{cm}^{2}\right)$ based on the DXA scans were obtained from the scan report. The vast majority of the DXA scans were performed with the same scanner at Vejle Hospital.

Statistical analyses. Categorical data were described with frequency and percentage. Continuous data were described using mean and standard deviations.

For the uni- and multivariate analyses all exposure variables were transformed into dichotomous data: ER-status (negative vs. positive), BMI (<25 vs. $>25$ ), smoking status (current smokers vs. former/never/unknown.), zoledronic acid (no vs. 


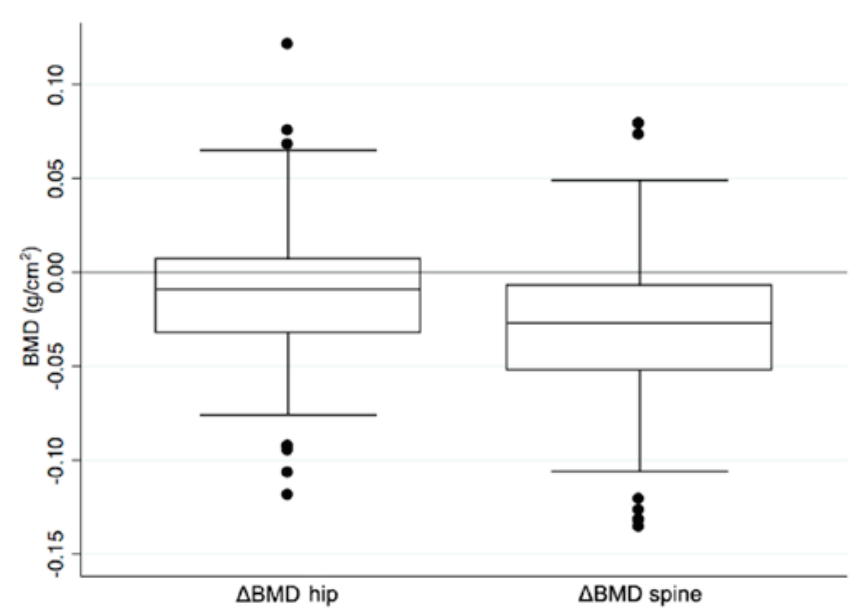

Figure 2. Boxplot of change in BMD from start of chemotherapy to one year later showing the interquartile range, median, and range with outliers.

yes), endocrine treatment (AI treatment vs. tamoxifen/none). The time from the last cycle of chemotherapy to the follow-up DXA scan was dichotomised by separating the observations at the median to evaluate whether those with a late follow-up DXA scan lost more BMD than the group with an early DXA scan.

The BMD measures in the lumbar spine and hip before chemotherapy and one year after as well as the difference between the first and second DXA scan of the hip and lumbar spine ( $\triangle B M D$ hip and $\triangle B M D$ spine) were tested for normality using QQ plots, which showed normality to be a reasonable approximation for the distributions. The paired t-test was used to test the null-hypothesis: No difference in BMD between the first and second DXA-scan of hip and lumbar spine, respectively.

In the univariate analyses, we used linear regression to test the association between $\triangle B M D$ hip and $\triangle B M D$ spine by different variables. When the association showed a P-value $<0.2$, the variable was introduced into the multiple linear regression analysis, which was performed to study the association between a variable and the BMD change, having controlled for the effects of other variables.

The analyses described were all planned in advance. All statistical analyses were carried out using STATA 14.2 for Mac (StataCorp Stata 14.2, College Station, TX, USA).

\section{Results}

Patient characteristics. The final analysis included 152 patients (Fig. 1). Patient characteristics are outlined in Table I showing that 79 patients $(52 \%)$ were premenopausal and 73 patients $(48 \%)$ postmenopausal. The mean age was 53 years (range 30 to 84 years). A total of 81 patients $(53 \%)$ had neoadjuvant chemotherapy and 71 patients $(47 \%)$ had adjuvant chemotherapy. At baseline 58 patients $(38 \%)$ had osteopenia and the remaining had a normal T-score $(>-1)$. The mean BMD at baseline was $0.933 \mathrm{~g} / \mathrm{cm}^{2}$ (95\% CI: 0.913; 0.952) and $1.014 \mathrm{~g} / \mathrm{cm}^{2}$ (95\% CI: 0.992; 1.036) for hip and lumbar spine, respectively. Thirty-one postmenopausal patients $(20 \%)$ had zoledronic acid treatment, 28 of which were treated once and three had zoledronic acid twice in the observation period.
Table I. Characteristics of the study population.

\begin{tabular}{|c|c|}
\hline & Study cohort $n=152$ \\
\hline Age at first DXA scan, years & Number $(\%)$ \\
\hline $30-39$ & $16(11)$ \\
\hline $40-49$ & $46(30)$ \\
\hline $50-59$ & $51(34)$ \\
\hline $60-69$ & $30(20)$ \\
\hline$>70$ & $9(6)$ \\
\hline \multicolumn{2}{|l|}{ Type of surgery } \\
\hline Lumpectomy & $107(70)$ \\
\hline Mastectomy & $45(30)$ \\
\hline \multicolumn{2}{|l|}{ Type of carcinoma } \\
\hline Ductal & $121(80)$ \\
\hline Lobular & $11(7)$ \\
\hline Other/unknown & $20(13)$ \\
\hline \multicolumn{2}{|l|}{ Oestrogen receptor status } \\
\hline Negative & $37(24)$ \\
\hline Positive & $115(76)$ \\
\hline \multicolumn{2}{|l|}{ HER2 status } \\
\hline Positive & $32(21)$ \\
\hline Negative & $119(78)$ \\
\hline Unknown & $1(1)$ \\
\hline \multicolumn{2}{|l|}{ Nodal status } \\
\hline 0 & $91(60)$ \\
\hline $1-4$ & $44(29)$ \\
\hline$>4$ & $17(11)$ \\
\hline \multicolumn{2}{|l|}{ Menopausal status } \\
\hline Premenopausal & $79(52)$ \\
\hline Postmenopausal & $73(48)$ \\
\hline \multicolumn{2}{|l|}{$\mathrm{BMI}$} \\
\hline$<25$ & $78(51)$ \\
\hline $25-30$ & $42(28)$ \\
\hline$>30$ & $32(21)$ \\
\hline \multicolumn{2}{|l|}{ Smoking status } \\
\hline Never & $79(52)$ \\
\hline Former smoker & $39(26)$ \\
\hline Current smoker & $33(22)$ \\
\hline Unknown & $1(1)$ \\
\hline \multicolumn{2}{|l|}{ Zoledronic acid } \\
\hline Yes & $31(20)$ \\
\hline No & $121(80)$ \\
\hline \multicolumn{2}{|l|}{ Endocrine treatment } \\
\hline Tamoxifen & $62(41)$ \\
\hline Aromatase inhibitor & $54(36)$ \\
\hline None & $36(24)$ \\
\hline \multicolumn{2}{|l|}{ Chemotherapy } \\
\hline Neoadjuvant & $81(53)$ \\
\hline Adjuvant & $71(47)$ \\
\hline
\end{tabular}




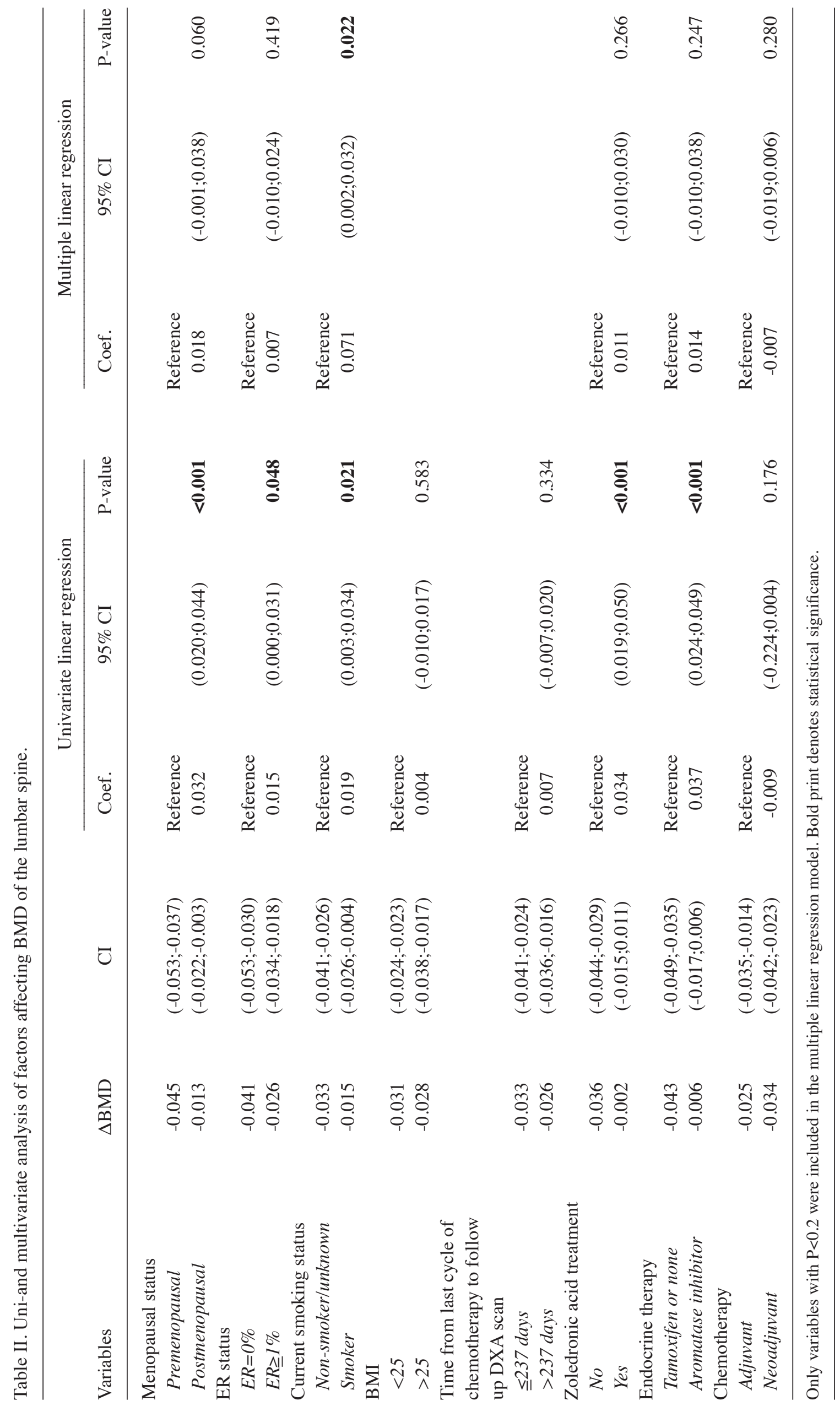


Overall changes in BMD. Patients receiving neoadjuvant/adjuvant chemotherapy had a significant loss in mean BMD. The mean change in BMD was $-0.0124 \mathrm{~g} / \mathrm{cm}^{2}$ (95\% CI -0.018; $-0.007 \mathrm{P}<0.001)$ in the hip and $-0.029 \mathrm{~g} / \mathrm{cm}^{2}$ (95\% CI: -0.036 ; $-0.023 \mathrm{P}<0.001)$ in the lumbar spine corresponding to a reduction in BMD of 1.3 and $2.9 \%$ for the hip and lumbar spine, respectively. Range and interquartile for BMD changes in spine and hip are shown in Fig. 2.

Univariate and multivariate analysis of factors affecting $B M D$. The results of the univariate and multivariate analyses of the lumbar spine BMD values are shown in Table II. In the univariate analysis the premenopausal patients had a substantial BMD loss of $0.045 \mathrm{~g} / \mathrm{cm}^{2}$ equivalent to $4.3 \%$. This was significantly higher than the loss seen in postmenopausal women $(\mathrm{P}<0.001)$. Furthermore, ER-positive patients had a lower BMD loss than ER-negative patients $(\mathrm{P}=0.048)$ as did current smokers compared to former smokers/unknown $(\mathrm{P}=0.021)$. Patients receiving zoledronic acid had a lower BMD loss than those who did not have zolendronic acid $(\mathrm{P}<0.001)$, and the BMD loss of patients treated with AI was lower than those treated with tamoxifen or having no endocrine treatment $(\mathrm{P}<0.001)$.

The multiple linear regression showed a lower BMD loss in current smokers than in non-smokers/unknown $(\mathrm{P}=0.022)$. No other variables in relation to the spine were statistically significant. There was a trend of higher BMD loss in premenopausal women compared to postmenopausal women $(\mathrm{P}=0.060)$.

In the univariate analysis of the BMD values from the hip (data not shown) a significantly larger BMD loss was found in the group with a BMI $<25$ in comparison to those with a $\mathrm{BMI}>25(\mathrm{P}=0.034)$. No other variables in this context showed statistical significance. When controlling for other variables in the multiple linear regression the higher extent of bone loss in the group with $\mathrm{BMI}<25$ compared to $\mathrm{BMI}>25$ persisted in the analysis of the BMD values on the hip $(\mathrm{P}=0.048)$. Zoledronic acid prevented bone loss in the lumbar spine in the univariate analysis $(\mathrm{P}<0.001)$, but the effect did not persist in the multivariate analysis $(\mathrm{P}=0.266)$. No significant effect of zoledronic acid was observed in the hip (univariate: $\mathrm{P}=0.162$, multivariate: $\mathrm{P}=0.826)$.

There was no significant difference in BMD loss between patients having adjuvant and neoadjuvant treatment in neither hip (univariate: $\mathrm{P}=0.667$ ) nor lumbar spine (univariate: $\mathrm{P}=0.176$, multivariate: $\mathrm{P}=0.280$ ).

\section{Discussion}

The main finding in this study is that early stage BC patients treated with chemotherapy had a significant loss of BMD in both hip $(-1.3 \%)$ and lumbar spine $(-2.9 \%)(\mathrm{P}<0.0001$ for hip as well as lumbar spine), which is in line with other studies $(10,20)$. Furthermore, in the univariate analysis premenopausal women had a significantly larger loss of BMD in the lumbar spine than postmenopausal women $(\mathrm{P}<0.001)$, though a trend only persisted in the multiple linear regression $(\mathrm{P}=0.060)$.

Other studies on premenopausal patients have found a range of BMD loss similar to ours (21). A bone loss of approximately $0.1 \mathrm{~g} / \mathrm{cm}^{2}$ corresponds to one standard deviation, and a loss of that size in the lumbar spine increases the risk of vertebral fracture by a factor of $2.3(22,23)$. Looking at the subgroup of premenopausal women with a BMD loss of $0.045 \mathrm{~g} / \mathrm{cm}^{2}$ after one year, this can translate into a considerably increased risk of fracture, if not countered with prophylaxis medication. These findings are important because the majority of premenopausal women develops premature ovarian dysfunction as a direct consequence of chemotherapy, leading to a decreased systemic oestrogen level and thereby further bone loss in the years to come $(18,21,24)$.

Corticosteroids are known to induce bone loss, but patients receiving neoadjuvant chemotherapy did not show a larger BMD loss than those receiving adjuvant chemotherapy, although they had two more cycles of chemotherapy and a higher total dose of corticosteroids.

The strength of this study is the relatively large number of patients undergoing very uniform antineoplastic treatment and the precise data on BMD change, since each patient was her own control. On the other hand, the retrospective design is a limitation, as it makes room for selection bias. The lack of detailed information on smoking, alcohol, physical activity, and level of vitamin D, which are all known to influence bone turnover, may be potential confounders. DXA scans measure the bone density but do not reflect bone quality.

Thirty-one patients received zoledronic acid as part of their anti-cancer treatment, which, however, did not change their outcome significantly in comparison to those, who did not receive zoledronic acid. This is probably due to the fact that the first dose was often given just before the follow-up DXA scan, and therefore, the inhibitory effect on osteoclasts resorption in the bone tissue did not have sufficient time to make a difference. In contrast, a randomized study from 2008, where $4 \mathrm{mg}$ zoledronic acid was given every three months vs. placebo, showed that zoledronic acid prevented BMD loss one year after chemotherapy (25).

The follow-up time in our study was between 9 and 15 months, and the loss of BMD did not change significantly with the length of follow-up. This indicates the majority of BMD loss to take place well before the time of follow-up.

Postmenopausal patients are now routinely offered zoledronic acid as part of the adjuvant treatment, since it has been shown to improve survival and reduce the risk of recurrence and bone fractures (19). Our results indicate that premenopausal women are likely to have accelerated bone loss, and it is therefore worth considering whether zoledronic acid should also be given to premenopausal women to reduce potential, long term side-effects.

In conclusion, this study confirms that neoadjuvant/adjuvant chemotherapy is associated with significant BMD loss in both the hip and lumbar spine. Furthermore, a pronounced loss of BMD in the lumbar spine in premenopausal women is suggested. Further studies with long term follow-up on bone loss and fracture risk evaluating prophylactic zoledronic acid in premenopausal patients are warranted.

\section{Acknowledgements}

The authors would like to thank René Depont Christensen, RD Statistics, for statistical assistance. 


\section{Funding}

No funding received.

\section{Availability of data and materials}

The analysed data sets generated during the study are available from the corresponding author on reasonable request.

\section{Authors' contributions}

The study was planned in collaboration between all authors. CA collected the data from the source. The primary analysis was made by $\mathrm{TB}$ and $\mathrm{CA}$ and the results were interpreted by $\mathrm{CA}, \mathrm{AJ}, \mathrm{EJ}$ and TB in collaboration. CA wrote the first draft for the manuscript. All authors revised it critically for important intellectual content and made changes in the iterative process that followed. This final manuscript has been approved by all authors.

\section{Ethics approval and consent to participate}

Data was collected through the electronic patient record system after approval by the Danish Health and Medicines Authority and the Danish Data Protection Agency. According to Danish law this study did not require approval by the Ethics Committee (project-ID: S-20150139).

\section{Consent for publication}

Not applicable.

\section{Competing interests}

The authors declare that they have no competing interests.

\section{References}

1. Ferlay J, Soerjomataram I, Dikshit R, Eser S, Mathers C, Rebelo M, Parkin DM, Forman D and Bray F: Cancer incidence and mortality worldwide: Sources, methods and major patterns in GLOBOCAN 2012. Int J Cancer 136: E359-E386, 2015.

2. Engholm G,Ferlay J, Christensen N,Kejs AMT,Hertzum-Larsen R, Johannesen TB, Khan S, Leinonen MK, Ólafsdóttir E, Petersen T, et al: 2016 NORDCAN: Cancer Incidence, Mortality, Prevalence and Survival in the Nordic Countries, Version 7.3 (08.07.2016). Association of the Nordic Cancer Registries. Danish Cancer Society. http://www.ancr.nu. Accessed March 19, 2017.

3. Hadji P: Cancer treatment-induced bone loss in women with breast cancer. Bonekey Rep 4: 692, 2015.

4. Hernlund E, Svedbom A, Ivergård M, Compston J, Cooper C, Stenmark J, McCloskey EV, Jönsson B and Kanis JA: Osteoporosis in the European Union: Medical management, epidemiology and economic burden. A report prepared in collaboration with the International Osteoporosis Foundation (IOF) and the European Federation of Pharmaceutical Industry Associations (EFPIA) Arch Osteoporos 8: 136, 2013

5. Cameron DA, Douglas S, Brown JE and Anderson RA: Bone mineral density loss during adjuvant chemotherapy in pre-menopausal women with early breast cancer: Is it dependent on oestrogen deficiency? Breast Cancer Res Treat 123: 805-814, 2010.
6. Hershman DL, McMahon DJ, Crew KD, Shao T, Cremers S, Brafman L, Awad D and Shane E: Prevention of bone loss by zoledronic acid in premenopausal women undergoing adjuvant chemotherapy persist up to one year following discontinuing treatment. J Clin Endocrinol Metab 95: 559-566, 2010.

7. Canalis E, Mazziotti G, Giustina A and Bilezikian JP: Glucocorticoid-induced osteoporosis: Pathophysiology and therapy. Osteoporos Int 18: 1319-1328, 2007.

8. Christensen CØ, Cronin-Fenton D, Frøslev T, Hermann AP and Ewertz M: Change in bone mineral density during adjuvant chemotherapy for early-stage breast cancer. Support Care Cancer 24: 4229-4236, 2016.

9. KalderMandHadji P:Breastcancerand osteoporosis-management of cancer treatment-induced bone loss in postmenopausal women with breast cancer. Breast Care (Basel) 9: 312-317, 2014.

10. Greep NC, Giuliano AE, Hansen NM, Taketani T, Wang HJ and Singer FR: The effects of adjuvant chemotherapy on bone density in postmenopausal women with early breast cancer. Am J Med 114: 653-659, 2003.

11. Bjarnason NH, Hitz M, Jorgensen NR and Vestergaard P: Adverse bone effects during pharmacological breast cancer therapy. Acta Oncol 47: 747-754, 2008.

12. Chen Y, Xu G and Yang F: Effect of neoadjuvant chemotherapy on the serum levels of bone turnover markers in women with early-stage breast cancer. PLoS One 10: e0126053, 2015.

13. Hadji P: Aromatase inhibitor-associated bone loss in breast cancer patients is distinct from postmenopausal osteoporosis. Crit Rev Oncol Hematol 69: 73-82, 2009.

14. Eastell R, Adams JE, Coleman RE, Howell A, Hannon RA, Cuzick J, Mackey JR, Beckmann MW and Clack G: Effect of anastrozole on bone mineral density: 5-year results from the anastrozole, tamoxifen, alone or in combination trial 18233230 . J Clin Oncol 26: 1051-1057, 2008.

15. Kanis JA, McCloskey EV, Powles T, Paterson AH, Ashley S and Spector T: A high incidence of vertebral fracture in women with breast cancer. Br J Cancer 79: 1179-1181, 1999.

16. Love RR, Mazess RB, Barden HS, Epstein S, Newcomb PA, Jordan VC, Carbone PP and DeMets DL: Effects of tamoxifen on bone mineral density in postmenopausal women with breast cancer. N Engl J Med 326: 852-856, 1992.

17. Yoneda K, Tanji Y, Ikeda N, Miyoshi Y, Taguchi T, Tamaki Y and Noguchi S: Influence of adjuvant tamoxifen treatment on bone mineral density and bone turnover markers in postmenopausal breast cancer patients in Japan. Cancer Lett 186: 223-230, 2002.

18. Vehmanen L, Elomaa I, Blomqvist C and Saarto T: Tamoxifen treatment after adjuvant chemotherapy has opposite effects on bone mineral density in premenopausal patients depending on menstrual status. J Clin Oncol 24: 675-680, 2006.

19. Early Breast Cancer Trialists' Collaborative Group (EBCTCG): Adjuvant bisphosphonate treatment in early breast cancer: Meta-analyses of individual patient data from randomised trials. Lancet 386: 1353-1361, 2015.

20. Shapiro CL, Manola J and Leboff M: Ovarian failure after adjuvant chemotherapy is associated with rapid bone loss in women with early-stage breast cancer. J Clin Oncol 19: 3306-3311, 2001

21. Hadji P, Gnant M, Body JJ, Bundred NJ, Brufsky A, Coleman RE, Guise TA, Lipton A and Aapro MS: Cancer treatment-induced bone loss in premenopausal women: A need for therapeutic intervention? Cancer Treat Rev 38: 798-806, 2012.

22. Garg MK and Kharb S: Dual energy X-ray absorptiometry: Pitfalls in measurement and interpretation of bone mineral density. Indian J Endocrinol Metab 17: 203-210, 2013.

23. Marshall D, Johnell O and Wedel H: Meta-analysis of how well measures of bone mineral density predict occurrence of osteoporotic fractures. BMJ 312: 1254-1259, 1996.

24. Riggs BL, Khosla S and Melton LJ III: Sex steroids and the construction and conservation of the adult skeleton. Endocr Rev 23: 279-302, 2002.

25. Hershman DL, McMahon DJ, Crew KD, Cremers S, Irani D, Cucchiara G, Brafman L and Shane E: Zoledronic acid prevents bone loss in premenopausal women undergoing adjuvant chemotherapy for early-stage breast cancer. J Clin Oncol 26: 4739-4745, 2008 . 